\section{Case Reports in Ophthalmology}

\title{
Spironolactone as an Adjunctive Treatment in Neovascular Age-Related Macular Degeneration
}

\author{
Kapil G. Kapoor Jennifer Sim \\ Eastern Virginia Medical School, Wagner Macula \& Retina Center, Virginia Beach, VA, USA
}

\section{Keywords}

Central serous chorioretinopathy - Neovascular age-related macular degeneration . Mineralocorticoid receptor antagonists · Eplerenone - Spironolactone

\begin{abstract}
Neovascular age-related macular degeneration (AMD) is a potentially sight-threatening condition. The current standard-of-care treatment regimen is serial intravitreal antivascular endothelial growth factor injections. While these typically have great success, they do carry exceptional treatment burden on the patient, cost burden due to their required frequency of use, and the risk of endophthalmitis, which can be devastating. This case report explores an alternative potential option as a treatment adjunct for neovascular AMD (nAMD), and identifies some of the overlap between nAMD and central serous chorioretionpathy. Future research is needed to better understand the role of mineralocorticoid receptor antagonist treatment in this disease spectrum.

(C) 2017 The Author(s)

Published by S. Karger AG, Basel
\end{abstract}

\section{Introduction}

Age-related macular degeneration (AMD) is the leading cause of vision impairment in subjects aged $>65$ years in the United States and the developed world [1]. Neovascular AMD (nAMD) can progress rapidly and may result in sudden vision loss caused by subretinal 
hemorrhaging and/or exudation of choroidal neovascularization (CNV) [1, 2]. While nAMD is typically a straightforward clinical diagnosis, sometimes a diagnostic dilemma can exist with subtypes of nAMD and other macular pathologies such as central serous chorioretinopathy (CSCR) [3]. CSCR is typically less likely to be associated with intraretinal fluid, subretinal hemorrhage, or exudates, and is more likely to present with isolated subretinal fluid (SRF) with a pachychoroid, and often in the setting of previous or current oral corticosteroid exposure [4].

The mainstay of therapy for nAMD is intravitreal antivascular endothelial growth factor (anti-VEGF). Though highly effective, $25-35 \%$ of the patients still have active exudation after 1 year of therapy (treatment-resistant nAMD), and at least $5 \%$ are persistently refractory [4]. While the clinical impact on patients and their vision from this is tremendous, this is also increasing the direct and indirect health care cost [4, 5]. In 2011, the direct annual cost per patient with nAMD (USD 34,308) was twice that of individuals without AMD (USD 17,473) [6]. The combined Medicare Part B expenditures of USD 2 billion for ranibizumab and bevacizumab in current nAMD treatment options will be progressively difficult to sustain as the aging baby boomer population will increase the prevalence of AMD from 2.07 million in 2010 to 5.44 million by 2050 in the United States alone according to the National Eye Institute (NEI) [7]. Practical factors of medication cost, reliance on caregivers, and the commitment of significant time and services from physicians, staff, and patients all are components of the significant treatment burden of nAMD.

As visual function declines with ongoing exudation, independence and overall quality of life decline equivalently [5]. nAMD increases the risk of depression, cognitive decline, and negatively impacts activities of daily living [5]. Further, 2 meta-analyses have reported a calculated pooled rate of endophthalmitis, a feared complication, after anti-VEGF injections at 52/105,536 injections $(0.049 \%)$ and $197 / 350,535$ injections $(0.056 \%)$ [8]. Since patients need repeated injections, the per-patient risk is greater than the per-injection risk [8]. Properly addressing potential adverse outcomes and the impact intravitreal injections can have on the patient's quality of life is an important consideration to take when selecting the ideal therapy for nAMD patients. Not only can previous experiences with endophthalmitis increase the emotional burden, but also the overall experience of intravitreal injections may result in frustration, anxiety, and needle phobia.

The vision morbidity with treatment-resistant nAMD and the burdens placed on patients who need frequent injections support the need to find alternative efficacious and costeffective treatment options for nAMD. Multiple alternatives have been explored, including higher anti-VEGF doses, alternating anti-VEGF agents, alternative medicinal options, and surgery intervention. In general, the limited evidence for these treatment alternatives is both variable and disappointing $[1,2,5]$.

One treatment option yet to be explored is mineralocorticoid receptor (MR) antagonists such as eplerenone and spironolactone. The eye has a dynamic aldosterone-MR system that plays an important pathological role in the development of angiogenic ocular conditions. The use of MR antagonists has been considered a promising treatment option for CSCR. However, to our knowledge, this is the first case report of spironolactone used to treat nAMD.

\section{Case Presentation}

A 74-year-old Caucasian female presented to our clinic with a 1-week history of blurred vision in the right eye. Her visual acuity at that visit was $20 / 50$ in the right eye. Optical co- 
herence tomography (OCT) macular examination revealed intact inner segment/outer segment layers and retinal elevation consistent with SRF, and the patient was slightly pachychoroid for her age (Fig. 1). Intravenous fluorescein angiography was suggestive of temporal leakage but equivocal. Her past medical history was remarkable for use of oral budesonide 3 $\mathrm{mg}$ for autoimmune hepatitis and loss of functional vision in the left eye after a remote history of endophthalmitis from intravitreal injections for nAMD in the left eye.

In concordance with the above findings, a diagnosis of acute CSCR secondary to her oral steroids was favored, although nAMD remained in the differential diagnosis, particularly given her contralateral eye history. An initial discussion with her gastroenterologist suggested that the budesonide was critical for her to continue. Given the potential for sightthreatening nAMD, she was advised to commence intravitreal anti-VEGF in the right eye, but adamantly refused based on fears rooted in her history of remote endophthalmitis in the contralateral eye. A course of oral spironolactone $25 \mathrm{mg}$ b.i.d. was initiated for presumed CSCR secondary to her oral steroids. We discussed that if this was refractory, she may benefit from photodynamic therapy if the condition became chronic. She was advised to monitor her Amsler grid daily and to attend a follow-up visit 2 weeks later.

On treatment day 14, her visual acuity improved to 20/40, and her OCT revealed decreased SRF (Fig. 2). On treatment day 28, visual acuity was stable at 20/40, and her OCT revealed complete resolution of the SRF (Fig. 3). However, on examination of the fundus, a temporal subretinal hemorrhage was present. Repeat fluorescein angiography was done and demonstrated clear leakage from a choroidal neovascular membrane consistent with nAMD (Fig. 4). This pattern suggested that a CNV was present the entire time, and spironolactone was successful in treating her SRF despite this. She was started on ranibizumab $0.5 \mathrm{mg}$ monthly intravitreal injections and continued spironolactone. At 2 years of treatment, there are no signs of endophthalmitis, and OCT findings show completely resolved SRF and stable nAMD. She has been gradually weaned off spironolactone, and her anti-VEGF regimen has been extended to once every 12 weeks (Fig. 5).

\section{Conclusion}

Central to CNV pathogenesis and changes in ocular structures seen in nAMD are ischemia, local inflammation, changes in vascular permeability, and growth factor-induced angiogenesis [9]. Among the numerous factors involved in the complex interplay in CNV, VEGF is the main stimulating factor for vascular permeability and angiogenesis [9]. However, as illustrated by this case report, anti-VEGF injections are not always favorable, and patients may be refractory to this treatment. VEGF is increased by other upstream factors such as TGF-b, IGF1 , and glucose, which are all modulated by the renin-angiotensin-aldosterone system (RAS) [10]. Subsequently, therapy that targets the RAS may be effective in treating retinal vasculopathy, especially because angiotensin II and aldosterone are proinflammatory, fibrogenic, and angiogenic [10].

In addition to the RAS components in the eye, the retina also possesses a functionally active aldosterone-MR system that plays an important pathological role in retinal vasculopathy [11]. Aldosterone activation of the MR directly stimulates neoangiogenesis to the extent of VEGF [11]. Corresponding clinical significance was reported when MR antagonism with spironolactone improved pathological angiogenesis most likely by suppressing the proinflammatory responses and vascular injury of reactive oxygen species [10]. MR recognized on the Müller glial cells of the neuroretina control potassium and water homeostasis via regulation 


\section{Case Reports in Ophthalmology}

of expression and distribution of ion and water channels [3]. Activation of these receptors is known to contribute to retinal edema [3]. Blocking proedematous consequences of MR activation is an alternative mechanism in which eplerenone and spironolactone may exert its effects.

Based on these preclinical results, MR antagonists were proposed as an option for CSCR treatment. Two interventional, uncontrolled, prospective case series tested either eplerenone or spironolactone in CSCR patients with SRF for at least 3 months [3]. In both studies, there was a significant decrease in SRF compared to baseline associated with an increase in visual acuity after 3 months of treatment [3]. A pilot study of patients with chronic CSCR of at least 4 months started on $25 \mathrm{mg}$ /day of eplerenone that was subsequently increased to $50 \mathrm{mg} /$ day were noted to have improvements in vision and OCT [12]. In a double-blinded, randomized control trial of spironolactone, treatment resulted in improved visual acuity and significantly decreased SRF and central macular thickness compared to placebo [13].

The current case suggests that oral MR antagonists may serve as an adjunctive treatment in nAMD, with emphasis in dehydration of SRF in the setting of chronic SRF. CSCR is associated with genetic variants (ARMS2 and $C F H$ ) also implicated in AMD, suggesting a genetic and pathophysiologic overlap between CSCR and AMD [3]. These indications are also suggested by findings in this clinical case as MR antagonists resolved SRF regardless of pathology. This case report supports the importance of correct clinical classification, and perhaps the reconstruction of the nAMD, polypoidal CNV, and CSCR spectrum [3]. Recently, this has become more pertinent as OCT allows for improved diagnosis and emergence of clinical subtypes such as type $1 \mathrm{CNV}$ developing in chronic CSCR that mimics nAMD [3].

Identification of MR antagonists as additional therapy may reduce patient injections and improve outcomes in refractory nAMD by targeting additional pathways. MR antagonists may inhibit CNV through a VEGF-independent pathway, which allows for treatment response potentiation or synergism when combined with anti-VEGF [14]. As physicians must constantly try to optimize patient outcomes and be mindful of looming budgetary limitations, this oral alternative may allow for the needed balance between patient quality of life, quality of care, and cost of care and reimbursements for provided time and services. Further research is needed to elucidate the exact role of oral MR antagonists in both CSCR and nAMD.

\section{Statement of Ethics}

The subject has given informed consent, and this research was approved by the institute's committee on human research.

\section{Disclosure Statement}

The authors have no disclosures.

\section{References}

Ambati J, Fowler BJ: Mechanisms of age-related macular degeneration. Neuron 2012;75:26-39.

Agarwal A, Rhoades WR, Hanout M, et al: Management of neovascular age-related macular degeneration: current state-of-the-art care for optimizing visual outcomes and therapies in development. Clin Ophthalmol 2015;9:1001-1015. 


\section{Case Reports in Ophthalmology}

\begin{tabular}{l|l}
\hline DOI: 10.1159/000475880 & $\begin{array}{l}\text { (c) 2017 The Author(s). Published by S. Karger AG, Basel } \\
\text { www.karger.com/cop }\end{array}$ \\
\hline
\end{tabular}
www.karger.com/cop

Kapoor and Sim: Spironolactone as an Adjunctive Treatment in Neovascular Age-Related Macular Degeneration

3 Daruich A, Matet A, Dirani A, et al: Central serous chorioretinopathy: recent findings and new physiopathology hypothesis. Prog Retin Eye Res 2015;48:82-118.

4 Broadhead GK, Hong T, Chang AA: Treating the untreatable patient: current options for the management of treatment-resistant neovascular age-related macular degeneration. Acta Ophthalmol 2014;92:713-723.

5 Prenner JL, Halperin LS, Rycroft C, et al: Disease burden in the treatment of age-related macular degeneration: findings from a time-and-motion study. Am J Ophthalmol 2015;160:725-731.

-6 Schmier JK, Covert DW, Lau EC: Patterns and costs associated with progression of age-related macular degeneration. Am J Ophthalmol 2012;154:675-681.

$7 \quad$ National Eye Institute: Age-related macular degeneration (AMD). Department of Health and Human Services. National Institutes of Health. https://nei.nih.gov/eyedata/amd\#1 (accessed February 2017).

-8 Merani R, Hunyor AP: Endophthalmitis following intravitreal anti-vascular endothelial growth factor (VEGF) injection: a comprehensive review. Int J Retin Vitr 2015;1:9-27.

-9 Nowak JZ: Age-related macular degeneration (AMD): pathogenesis and therapy. Pharmacol Rep 2006;58:353-363.

-10 Touyz RM, Callera GE: A new look at the eye: Aldosterone and mineralcorticoid receptors as novel targets in retinal vasculopathy. Circ Res 2009;104:9-11.

11 Wilkinson-Berka JL, Tan G, Jaworski K, et al: Identification of a retinal aldosterone system and the protective effects of mineralcorticoid receptor antagonism on retinal vascular pathology. Circ Res 2009;104:124-133.

$\$ 12$ Bousquet E, Beydoun T, Zhao M, et al: Mineralcorticoid receptor antagonism in the treatment of chronic central serous chorioretinopathy: a pilot study. Retina 2013;33:2096-2102.

13 Bousquet E, Beydoun T, Rothchild PR, et al: Spironolactone for non-resolving central serous chorioretinopathy: a randomized controlled cross-over study. Retina 2015;35:2505-2515.

14 Zhao M, L XX, Arboleda A, et al: Mineralcorticoid receptor antagonists exert anti-angiogenic effects in laser- induced choroidal neovascularization independently from VEGF pathway. Euretina (in press).

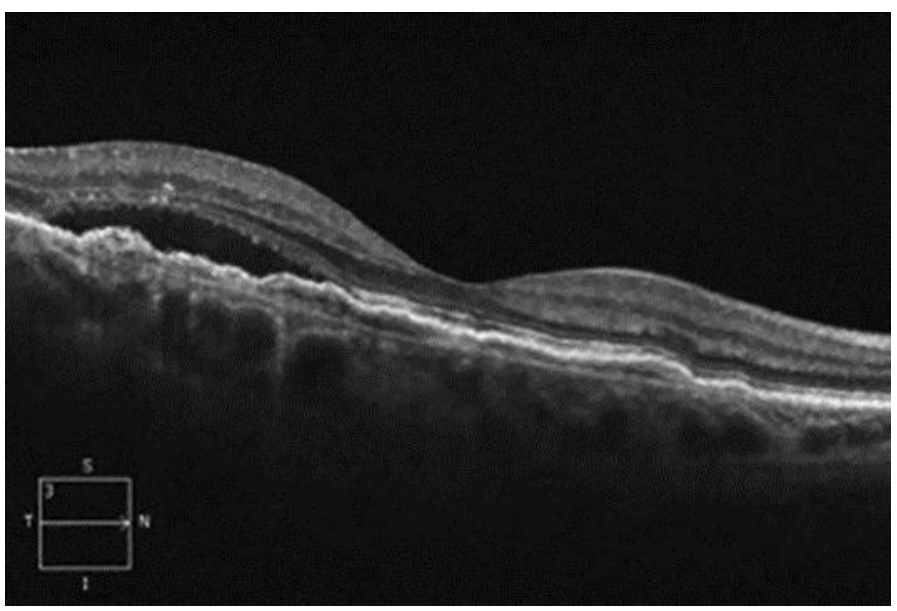

Fig. 1. Optical coherence tomography of day 0 treatment with spironolactone revealing intact inner segment/outer segment layers and retinal elevation consistent with subretinal fluid. 
Case Reports in
Ophthalmology

Case Rep Ophthalmol 2017;8:314-320

(c) 2017 The Author(s). Published by S. Karger AG, Basel www.karger.com/cop

Kapoor and Sim: Spironolactone as an Adjunctive Treatment in Neovascular Age-Related Macular Degeneration

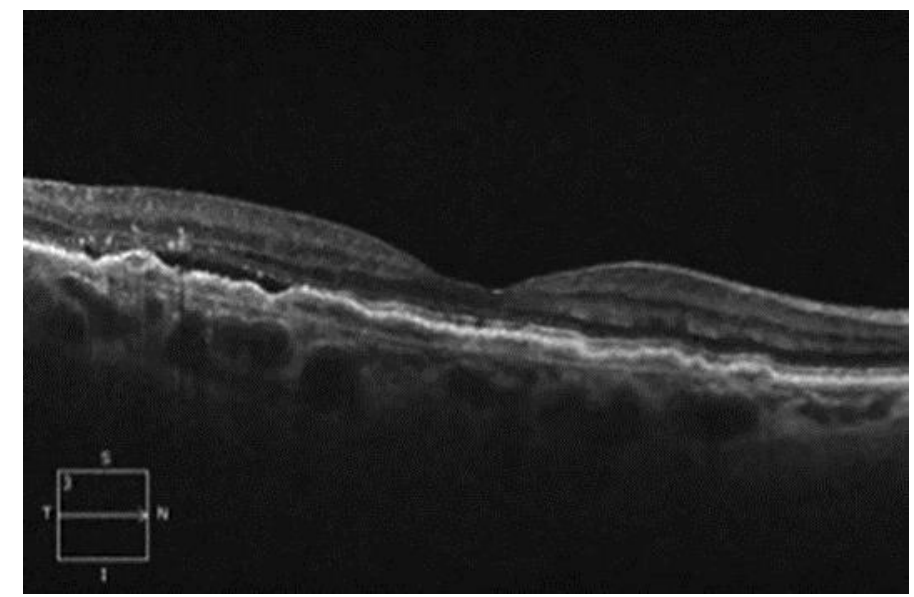

Fig. 2. Optical coherence tomography of day 14 treatment with spironolactone revealing decreased subretinal fluid.



Fig. 3. Optical coherence tomography of day 28 treatment with spironolactone revealing resolved subretinal fluid. 


\section{Case Reports in Ophthalmology} www.karger.com/cop

Kapoor and Sim: Spironolactone as an Adjunctive Treatment in Neovascular Age-Related Macular Degeneration

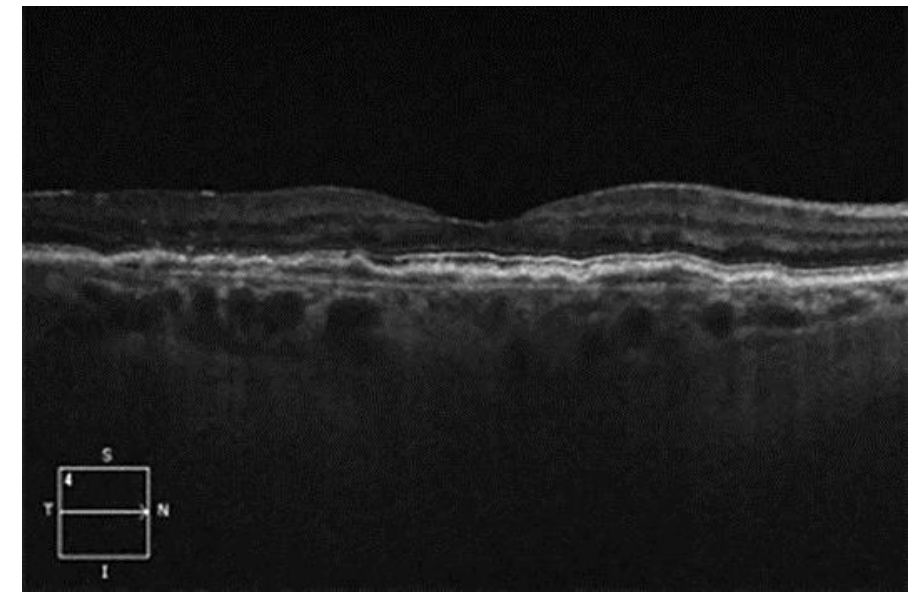

Fig. 4. Fluorescein angiography at day 28 revealing occult leakage from the choroidal neovascular membrane with associated blockage from a subretinal hemorrhage.

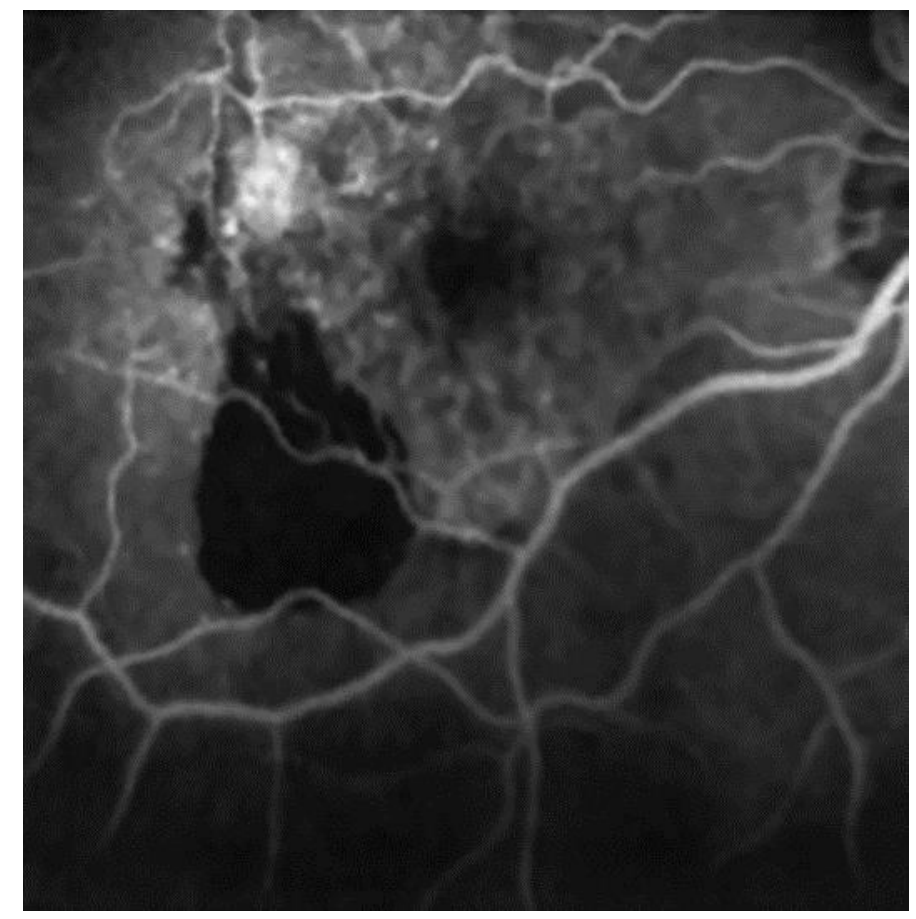

Fig. 5. Optical coherence tomography at 2 years revealing sustained resolution of the subretinal fluid. 